\title{
Carnets
}

Revue électronique d'études françaises de l'APEF

Deuxième série - 5 | 2015

Imaginaires de guerre et autres conflits

\section{Imaginaires de guerre. Claude Simon et Pascal Quignard}

Du vécu à l'écriture

Agnès Cousin de Ravel

\section{(2) OpenEdition}

Journals

Édition électronique

URL : http://journals.openedition.org/carnets/455

DOI : $10.4000 /$ carnets. 455

ISSN : 1646-7698

Éditeur

APEF

Référence électronique

Agnès Cousin de Ravel, «Imaginaires de guerre. Claude Simon et Pascal Quignard », Carnets [En ligne], Deuxième série - 5 | 2015, mis en ligne le 30 novembre 2015, consulté le 20 avril 2019. URL : http:// journals.openedition.org/carnets/455 ; DOI : 10.4000/carnets.455

Ce document a été généré automatiquement le 20 avril 2019.

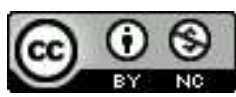

Carnets est mis à disposition selon les termes de la licence Creative Commons - Atribution - Pas d'utilisation commerciale 4.0 International. 


\title{
Imaginaires de guerre. Claude Simon et Pascal Quignard
}

\author{
Du vécu à l'écriture
}

Agnès Cousin de Ravel

127 août 1914 : Le capitaine Louis Simon est tué à la guerre d'une balle au front.

227 août 1939 : Son fils Claude est mobilisé. Il part en train vers le front des Ardennes et refait le trajet que son père avait fait vingt-cinq ans auparavant.

3 10-17 mai 1940 : Claude Simon est au cœur de la guerre. Son régiment de cavalerie, sabre au clair, est décimé par l'artillerie et l'aviation allemandes. Restent seulement quatre survivants dont lui.

423 avril 1948: Pascal Quignard naît à Verneuil-sur-Avre. La famille Quignard habite 3 Place Saint-Jean. Des fenêtres de la maison, on voit les ruines de l'église Saint-Jean bombardée par les Alliés en 1944.

5 Décembre 1951 : La famille Quignard emménage au 86 rue Bernardin de Saint-Pierre au Havre dans l'un des premiers immeubles Perret tout juste sortis de terre, sur le champ de ruines laissées après le bombardement du 4 septembre 1944 qui a détruit la ville à $80 \%$. De la fenêtre de sa chambre, l'enfant voit un bidonville. Quotidiennement, il traverse des champs de ruines pour se rendre au lycée et à ses leçons de solfège et de piano.

6 Ces quelques dates mettent en lumière deux des trois temps propres à la guerre : 1 . Celui de la destruction en acte, de la guerre dans son terrifiant accomplissement. C'est Simon. 2. Celui des ruines. C'est Quignard. 3. Celui de la reconstruction dont je ne parlerai pas. Ces deux premiers temps déploient deux «imaginaires» de la guerre (le mot " imaginaire » entendu comme modalités de représentation) bien différents chez Simon et chez Quignard, même si leurs propos sur la guerre se rejoignent, même si tous deux pratiquent un art certain de la fragmentation.

7 Peu ou prou, tous les romans de Simon, parlent de la guerre. Je pense sa représentation essentiellement à partir de quatre d'entre eux : La Route des Flandres (1960), L'Acacia (1989), Histoire (1967) et Le Jardin des Plantes (1997) et aussi et Quatre conférences (2012). Chez Quignard, la guerre est présente dans L'Occupation américaine (1994), La Haine de la musique 
(1995) et dans quelques-uns des tomes de son Dernier royaume, en particulier dans Les Ombres errantes (2002) et Les Désarçonnés (2012) ainsi que dans son tout récent Sur l'idée d'une communauté de solitaires (2015).

D'où trois directions : 1 . Les faits. Les représentations se fondent chez les deux écrivains sur des faits vécus et/ou racontés et/ou lus 2. Les faits induisent un discours démythifiant sur la guerre. 3. Ces faits, dans leur puissance destructrice, sont chez les deux écrivains, le substrat de leur écriture.

\section{Faits de guerre}

9 Chez Simon, la guerre telle qu'il l'a vécue, en a souffert, est essentiellement la Seconde Guerre mondiale, mais aussi la Première Guerre mondiale, avec, en arrière-plan les guerres révolutionnaires de "l'ancêtre », LSM (Lacombe Saint Michel). De La Route des Flandres au Jardin des Plantes, les faits sont de plus en plus précis. Dans les manuscrits ${ }^{1}$ de L'Acacia, trois souvenirs sont déterminants selon la chronologie propre à l'écriture. D'abord, la mort du père: «Le 27 août 1914 à la lisière d'un bois, près du village de Sterray, sur la Meuse, le capitaine $\mathrm{S}$. du $24^{\mathrm{e}}$ régiment d'Infanterie coloniale fut blessé par une balle et transporté par ses hommes jusqu'au pied d'un arbre contre lequel ils l'adossèrent (...). » (SMN 16 (1) 7/503). Puis la recherche des restes du père sur le champ de bataille quand il avait à peine six ans (la phrase manuscrite, presque inchangée, deviendra la première du roman) : «Elles allaient d'un village à l'autre, et dans chaque village (ou du moins ce qu'il en restait) d'une maison à l'autre » (SMN 16 (1) 8/503). Et enfin, le départ de son père à la guerre :

Départ de mon père (souligné en rouge)

Le 3 ou 4 août, dans l'après-midi, la famille réunie (...) De la chambre entendent les sabots du cheval (amené par l'ordonnance) sur le ciment (ou pavé) de la cour. Adieux (ajout : à tous) dans la véranda.

Mon père (...) Embrassant toute la famille. Ma mère à mon père: «À moi le dernier!»

Moi en bas (...) mon père m'embrassant une dernière fois avant de monter à cheval

(...) Tournant à droite dans la rue de la Cloche d'Or après avoir franchi le portail. »

(SMN 16 (1) 50/503 « 27 novembre $82 »)$

Les trois événements s'inscrivent dans les romans, sans ordre chronologique, dans la trame narrative comme des scènes revenantes. Le départ du père est développé d'abord dans le chapitre VII puis repris sous une forme condensée et métaphorisée dans le chapitre IX du roman :

(...) la séparation, le départ, le cheval dans la cour, l'homme harnaché pour la guerre, la famille au balcon de la véranda - et elle au premier rang, au milieu, comme dans une loge de théâtre, (...), regardant cette fois, pâle mais sans larmes, les mâchoires serrées, l'homme de guerre saisir l'enfant que lui tendait la négresse, l'embrasser, le presser un instant contre sa poitrine barrée de courroies, le rendre à la négresse, enfourcher le cheval, l'éperonner et disparaitre (...) (Simon, 2013b: 1176)

11 La mort du père est attestée dans des rapports militaires que Simon a consultés lors de ses recherches au SHAT de Vincennes (Service historique de l'Armée de terre). De même, il a eu communication des rapports concernant les événements de mai 1940 :

Le 16 mai à $11 \mathrm{~h} 30$, le capitaine A. de V... Cdt le $1^{\mathrm{e}}$ groupe de 105 modèle 1913 du $104^{\mathrm{e}}$ R.A.L.A. arrive au PC du $3^{\mathrm{e}}$ Bataillon du $84^{\mathrm{e}}$ R.I.F. à RAMOUSIES (chef de bataillon B...). (...) 15 heures - (..) 16 heures $45-($...) Il y a une autorité 
responsable, on n'envoie pas un groupe lourd au feu sans munitions, car ce manque de munitions a seul permis à une Panzer de percer la ligne à CLAIRFAYTS (...). (Simon, $2006: 1003$ ) six jours seulement après l'entrée du régiment en Belgique, le général qui commandait la brigade se fit sauter la cervelle.» (Simon, $2006: 1025)$ et Simon «vu» par Quignard: «Claude Simon se rendit à la guerre à cheval, sur le front belge. C'est le mois de mai 1940. Tenant les rênes dans sa main gauche, il reçoit l'ordre de brandir son sabre dans sa main droite contre les avions. » (Quignard, $2012: 30$ )

27 août 1914/27 août 1939. La mort s'inscrit dans la coïncidence des dates. La mort du père (son « destin » en quelque sorte noué dès l'entrée à Saint-Cyr) ne peut que prescrire celle du fils partant à la guerre (la mobilisation en 1939, la « drôle de guerre » jusqu'en mai 1940, le massacre puis le camp de prisonniers en Allemagne et la fuite). Les douze chapitres de L'Acacia sont ordonnés selon ces deux faits, ces deux temporalités. Dans le chapitre III « 27 août 1914 », la mort du père : « Parmi ceux qui tombèrent dans le combat du 27 août se trouvait un capitaine de quarante ans dont le corps encore chaud dut être abandonné au pied de l'arbre auquel on l'avait adossé. » (Simon, 2013 : 1043). Puis, à deux reprises, dans le chapitre " 27 août 1939 », la monstrueuse épée de Damoclès de la coïncidence des dates pour celui qui est désigné comme « (le brigadier) ». «Et maintenant il allait mourir.» (ibid. : 1108) Puis, vers la fin de ce chapitre : «Et maintenant tout cela était loin, fini, et il allait mourir (...)» (ibid.:1127). Son sort est scellé. Tout semble chez Simon se nouer là, se rapporter à ce coup du sort dont les romans seraient d'infinis développements, d'infinis ressassements, d'innombrables fragments. La Route des Flandres, L'Acacia et Le Jardin des Plantes semblent, au fond, ne dire que cela, revenir en boucle sur la mort du père et la déroute des Flandres.

Chez Quignard, pas de guerre au front (il a effectué son service militaire au Camp des Loges de Saint-Germain en Laye en tant que chauffeur du vaguemestre) mais le souvenir inscrit dans la mémoire familiale puisque chaque génération a eu un homme au front : « Il se trouve que mon père, mes deux grands-pères, la plupart de mes arrière-grands-pères ont combattu au cours des trois guerres franco-allemandes qui se sont succédé. Le 19 juillet 1870, le 3 août 1914, le 3 septembre 1939, (...).» (Quignard, 2012 : 16) Son grandpère Charles Bruneau engagé dans la Première Guerre mondiale « tint son journal intime en latin, dans sa tranchée, (...) au cœur de la guerre de 1914.» (ibid. : 90) Plus tard, Charles Bruneau dira à ses petits-enfants que la vie dans les tranchées avait été invivable, horrible, irracontable. Impossibilité de dire, perte du pouvoir parler Sprachlosigkeit: «Indicibilité de ce qui est vécu au front dans les mots - pour ne pas parler de la Propaganda qui a cours à l'arrière.» (Quignard, 2002: 115) Et plus prégnantes, plus déterminantes, deux images de l'enfance souvent revenantes : celle de son oncle Jean de retour de Dachau, toujours affamé, les poches pleines de bonbons, de bâtons de réglisse :

Il revint plus tard que Robert Antelme. Il se reconstitua plus lentement. D'une certaine manière il mit dix ans à revenir.

D'abord il fut - comme ils étaient toutes et tous - irreconnaissable. (...) Sans

cheveux.

Sans sourire. (...) Avec des yeux immenses.

La peau collant sur les os du squelette. (...) (Quignard, 2005 : 92-93)

et celle des lieux de l'enfance, à Verneuil puis au Havre le spectacle des ruines devenant socle de l'écriture dans une évocation très dense : 
J'ai vécu au Moyen-Âge.

J'ai appris à lire auprès d'un homme qui revenait du camp de Dachau. J'ai vécu enfant dans les ruines d'un port qui avait été entièrement bombardé, puis entièrement incendié lors d'un second largage de bombes.

Je roulais, sur un tricycle en fer, dans un square qui était le charnier de la ville où on avait enfoui les marins, les dockers, les pêcheurs, tous les chevaux de grève, tous les petits enfants des morts.

Mais tel était le rivage alors. Des ruines. Et telle est cette étrange rive que j'ai reconstituée dans les formes singulières des livres que j'ai imaginées. Non pas une terre, une demeure, une cité, un palais, un temple. Mais un rivage en ruines, un rivage désolé, plein de landes et d'épaves. (Quignard, 2015 : 33)

J'ajoute à ces ruines les vestiges de la langue allemande parlée par la jeune Caecilia Müller qui s'est occupée de lui pendant un an, alors qu'il était infans, non-parlant encore, langue complétement enfouie en lui, jusqu'à ce que son analyse avec le psychanalyse André Auscher la fasse revenir. J'ajoute enfin la représentation de l'exode chez les deux écrivains ; ces six semaines durant lesquelles la France s'est décomposée. Simon l'a vécu sur le champ de bataille :

(...) alors imaginez la calme fin d'une journée de printemps, (...) c'est le silence, personne ne parle, vous regardez seulement les premiers groupes de réfugiés qui cheminent en sens inverse, et tout d'un coup, (...) vous les entendez crier, (...) Qu'est-ce qui leur prend? qu'est-ce qui leur prend? en même temps que vous les voyez tous, femmes, hommes, enfants, abandonner les chariots, les bicyclettes ou les poussettes qu'ils traînaient et se jeter dans les fossés (...) (Simon, 2006 : 958-959)

Et Quignard dans la remémoration de l'exode enduré par sa mère : « Ma mère était seule sur les routes, âgée de vingt ans. Elle guidait ses grands-parents paternels. Elle les perdit sur la route. Ce fut le pire souvenir de sa vie. (...) Elle erra de Maurepas à Nontron, à Terrasson, au Blanc » (Quignard, 2013 : 111-112.)

\section{Démythification des valeurs}

17 Chez Simon et chez Quignard, ce vécu, cette mémoire et cette connaissance de la guerre n'en déploient aucune héroïsation, mais tous deux en déconstruisent le processus humain. Tous deux se rejoignent dans une pensée de la guerre comme intrinsèquement liée à l'humanité. En 1996, Quignard publie "Traité sur Esprit », longue préface au livre de Jacques Esprit contemporain de La Rochefoucauld, La Fausseté des vertus humaines ${ }^{2}$. Esprit démonte les vertus. Ce qui pousse les hommes à agir, ce ne sont pas le courage, l'honnêteté, l'honneur, la générosité mais des sentiments beaucoup moins valorisants aux yeux de soi et de la société, que sont la peur, l'appât du gain, la bassesse. Quignard, à sa suite, provoque : «Que voulez-vous dire par penchants moraux de l'espèce humaine (...) La guerre? Les camps polonais? Les camps de Sibérie? Les fosses du Rwanda? Les étagères métalliques du Cambodge ?» (Quignard, 2012 : 158).

18 La guerre défait les services publics qui structurent l'État (en arrière-fond du propos de Quignard: l'arrestation de son grand-père Charles Bruneau, alors Professeur à la Sorbonne, par la Gestapo en février 1942, sans doute sur dénonciation et la rafle du Vel d'Hiv., le 16 juillet 1942) : «La devise de ma famille aurait pu être : Méfie-toi de la SNCF. Méfie-toi de la RATP. Méfie-toi des gendarmes qui sonnent à ta porte.» (ibid. : 115) Pour Simon, la guerre de mai 1940 a détruit jusqu'aux valeurs propres à la guerre :

et alors quand il a appris, c'est-à-dire s'est rendu compte, a fini par comprendre que sa brigade n'existait plus, avait été non pas anéantie, détruite selon les lois - 
ou du moins ce qu'il pensait être les lois - de la guerre: normalement, correctement, comme, par exemple en montant à l'assaut d'une position imprenable, ou encore par un pilonnage d'artillerie, ou même encore - cela il l'eût peut-être à la rigueur admis - submergé par une attaque ennemie ; mais pour ainsi dire absorbée, diluée, dissoute, bue, effacée de la carte d'état-major sans qu'il sût où, ni comment ni à quel moment (...) (Simon, 2006 : 333)

De même, sur le plan individuel, l'héroïsme de la mort n'est qu'un maquillage La marche déterminée du colonel qui avance à la tête des lambeaux de son régiment, au mépris apparent de tout danger, n'est en fait que « ce suicide que la guerre lui donnait l'occasion de perpétrer d'une façon élégante (...), profitant en quelque sorte avec discrétion et opportunité de l'occasion offerte pour en finir avec ce qui n'aurait jamais dû commencer quatre ans auparavant. » (Simon, 2006 : 200) Comme en écho chez Quignard : « Mourir à la guerre est la mort 'culturelle' par excellence. » (2012 : 206) La guerre met à nu les ressorts des fonctionnements sociaux hors de toute morale. La guerre est plus féroce que la prédation animale car elle est guidée par la cruauté :

La chasse est excitante mais la guerre est passionnante. Elle rompt les limites. (...) . La guerre c'est l'âme de chacun en alerte, les classes d'âge devenues solidaires dans l'impatience de l'instant qui va suivre (...), le temps devenu unanime dans l'événement partageable par tout un chacun dans les «nouvelles » toujours neuves, dont la nouveauté se renouvelle sans cesse. C'est le tocsin. C'est le réveil en sursaut, les heures devenues substantielles, l'Histoire devenue signifiante. (ibid. :205)

Parce qu'elle est l'expression de la passion de la lutte au cœur du fonctionnement social, en temps de paix, elle a sa doublure économique :

l'homme ne connaissait que deux moyens de s'approprier ce qui appartient aux autres, la guerre et le commerce, et qu'il choisissait en général tout d'abord le premier parce qu'il lui paraissait le plus facile et le plus rapide et ensuite, mais seulement après avoir découvert les inconvénients et dangers du premier, le second c'est-à-dire le commerce qui était un moyen non moins déloyal et brutal mais plus confortable. (Simon, $2013: 215$ )

21 Écho chez Quignard évoquant La Part maudite de Georges Bataille. Enfin, dans la guerre la puissance destructrice du temps se ramasse. Il est «l'incohérent, nonchalant, impersonnel et destructeur travail du temps " (Simon, 2006: 412). Même écho chez Quignard: "À chaque déclaration de guerre (...) Temps qui tente une sortie du temps dans la mort non plus naturelle mais donnée. » (Quignard, 2012 : 209-210) De même dans les camps. Le compositeur Karel Fröhlich a composé dans le camp d'Auschwitz :

L'insécurité y était absolue, le lendemain était promis à la mort, l'art était la même chose que la survie, l'épreuve du temps avait à faire l'épreuve du passage du temps le plus interminable et le plus vide. Il ajoute que le public était aussitôt mort qu'il avait écouté. (Quignard, $2000: 232$ )

$\mathrm{Au}$ fond de la guerre, la pulsion de mort, selon l'expression inventée par Sabina Spielrein, fascine les humains. C'est pourquoi elle est sans fin, en soi comme au cœur de la société. À la question que Quignard pose: "Warum Krieg? Unde bella?", sa réponse empruntée à Sénèque (dans Hercule furieux) est lapidaire : «Bella delectat cruor. La guerre adore le sang. La destruction violente d'autres hommes plongea les hommes qui s'y livraient dans une excitation qui n'était pas comparable à celle qu'ils éprouvaient lors des prédations antérieures. » (Quignard, 2012 : 232) Le spectacle du sang versé a toujours fasciné et il fait des guerres « les fêtes sociales par excellence » (ibid. : 234) parce qu'elles sont l'expression de la prédation originaire que manifestent aujourd'hui encore «les fascismes, les religions, les nationalismes ». En résumé : 
L'histoire était une brève intrigue de temps à autre parricide, le plus souvent fratricide, qui se répétait sans finir, en hurlant à la mort. Ce n'étaient pas la liberté et la démocratie qui avaient triomphé des camps : c'étaient les camps nazis qui avaient contaminé l'ensemble de l'Europe. (Quignard, 1994 : 118)

\section{Comment dire l'indicible de la guerre?}

Comment dire? "Comment savoir?» demande Simon dans La Route des Flandres. Comment trouver les mots pour dire l'horreur de la guerre, la déshumanisation, la cruauté, la proximité de la mort, au-delà de la peur ? Comment ordonner dans le langage "le trouble magma d'émotions, de souvenirs, d'images qui se trouve en [lui]» (Simon, 2006 : 898). L'anéantissement de l'escadron peut-il être banalisé dans le langage au point de n'être qu'une « une affaire » comme elle l'est pour le capitaine : « exemple ne dites pas "l'escadron s'est fait massacrer dans une embuscade", mais "nous avons eu une chaude affaire à l'entrée du village de" (...) » (Simon, 2006 : 307) Quels mots trouver pour dire l'instant où tout se désagrège, quand l'obus pulvérise tout, quand la terre n'est plus que poussière, quand les sensations sont aux limites du sensible, aux limites du dire? Exemple : Le mot « obus» :

entre le lire dans les livres ou le voir artistiquement représenté dans les musées et le toucher et recevoir les éclaboussures c'est la même différence qui existe entre voir écrit le mot obus et se retrouver d'un instant à l'autre couché cramponné à la terre et la terre elle-même à la place du ciel et l'air lui-même qui dégringole autour de toi comme du ciment brisé des morceaux de vitres, et de la boue et de l'herbe à la place de la langue, et soi-même éparpillé, et mélangé à tellement de fragments de nuages, de cailloux, de feu, de noir, de bruit et de silence qu'à ce moment le mot obus et ou le mot explosion n'existe pas plus que le mot terre, ou ciel, ou feu, ce qui fait qu'il n'est pas possible de raconter ce genre de choses qu'il n'est pas possible de les éprouver de nouveau après coup, et pourtant tu ne disposes que de mots, alors tout ce que tu peux essayer de faire... (Simon, $2013: 244$ ).

Autant dans La Route des Flandres que dans L'Acacia et dans Le Jardin des Plantes, Simon contient la toute-puissance des scènes cruciales inscrites en lettres de sang dans sa mémoire (l'attaque par les Allemands, la déroute, les quatre cavaliers errants avec à leur tête un capitaine muet comme un mort-vivant, le voyage vers le camp de prisonniers dans le wagon, le camp de prisonniers) en les inscrivant dans la temporalité narrative disloquée, fragmentée, hors de la chronologie de l'émotion. Simon lu par Quignard: «Tout est pulsion revenante, marée revenant, marée interrogative, vague inachevable qui se soulève et qui avance et qui déborde, imposant chaque fois sa forme différente. » ( in Calle-Gruber, 2009: 209) Ainsi, dans L'Acacia, la dé-construction du temps, sa fragmentation est renforcée par le plan général du roman et sa composition interne en plans qui se nouent, se croisent, s'entrechoquent alors que dans Le Jardin des Plantes, le dialogue de $\mathrm{S}$. avec le journaliste est la ligne mélodique sur laquelle viennent s'inscrire des scènes, des événements plus ou moins lointains, rattachés ou non à la guerre. Enfin, dans La Route des Flandres, la trame narrative n'est jamais induite par une quelconque chronologie. Bien au contraire, elle est constamment subvertie par la succession de plans (comme autant de réitération) et par des dialogues renforçant l'effet (entre Georges et Blum par exemple). Participent également à la déconstruction chronologique les déplacements successifs, les réseaux métaphoriques montrant, par exemple, ce que devient l'homme soumis à la violence de la guerre. Ainsi, l'individu transporté dans des wagons à bestiaux et comme des bestiaux, ne vaut pas davantage que du « fret de retour 
des caisses vides ou du matériel avarié» (Simon, 2006: 243). Il n'est que la partie négligeable d'un troupeau « une troupe peut se muer en quelques instants en un troupeau détalant et affolé » (ibid. : 326) ; et le champ de bataille jonché de morts n'a pas l'odeur d'un charnier mais celle d'un " tas d'ordures, simplement puant, comme peut puer un tas de vieilles boîtes de conserves, d'épluchures de légumes et de chiffons brûlés, et pas plus émouvant ou tragique qu'un tas d'ordures » (ibid.: 334). Dans la guerre, l'humain se résorbe dans le néant :

mais comment appeler cela : non pas la guerre non pas la classique destruction ou extermination d'une des deux armées mais plutôt la disparition l'absorption par le néant ou le tout originel de ce qui une semaine auparavant était encore des régiments des batteries des escadrons des escouades des hommes (ibid.: 275)

Cette désintégration sociale, individuelle - premier renversement à l'échelle humaine de tout ce qui jusque-là a paru faire croire en un progrès de l'humanité - et la violence du vécu ne peuvent se dire que dans une langue qui prend en charge la subversion de ses propres codes, dans une forme d'éclatement du dire. Si Simon a pris soin de ne pas être affilié au Nouveau Roman (de ne pas en devenir l'affidé), de La Route des Flandres au Jardin des Plantes, il déploie avec toujours plus de sureté, de maîtrise (comme en témoignent les feuillets manuscrits des plans de ses romans) un système rigoureux de dé-construction des représentations, non dans quelque souci d'originalité, mais pour retrouver dans le langage le chaos de ses sensations et de ses émotions. Il obéit en cela à la nécessité que (selon Quignard évoquant l'entretien de Merleau-Ponty avec Claude Simon): «la fragmentation soit cut, incomplétude, inencrantable, irremplissable, différence abrupte, vide. » (Quignard in Calle-Gruber, 2014 : 300) Exemple : Dans Le Jardin des Plantes, la guerre s'inscrit dans l'entrechoc de fragments souvent datés (10 mai 1940 à cinq heures du matin, 17 mai 1940 par exemple.) mais disposés dans le roman sans aucun ordre chronologique, mentionnant des lieux géographiquement repérables (la frontière entre la Belgique et la France, la Meuse, Solre-le Château où ont eu lieu les attaques de l'armée allemande sous la direction de Rommel rapportant lui-même son action et le mouvement de ses troupes dans de brèves lettres à sa femme. Ces fragments, éclatés dans tout le roman, sont en tension, en résonance aussi, avec d'autres fragments remémorant, entre autres, des bribes de la vie de son père (les cartes postales de pays lointains, l'Égypte, l'Inde, que Louis Simon envoyait à sa fiancée), sa mère sur son lit de mort, des séjours que lui-même a faits (en URSS, à Tokyo, à New-York), ou encore le Jardin des plantes à Paris et les pays de son enfance, le Sud chaud et lumineux, le Jura très vert. Ils donnent plus de force encore à l'inscription du peintre Gastone Novelli avec 1 . La description de quelquesuns des tableaux de ce dernier, 2. Son séjour au Brésil dans la forêt amazonienne après la guerre, après avoir été torturé par les Allemands, parce que « non seulement il ne pouvait plus supporter la vue d'un Allemand ou d'un uniforme mais même celle d'un être dit civilisé » (Simon, 2006 : 913). 3. À Dachau, là où comme dans tous les camps, la menace de mort, pour un rien, était constante, des «scènes " très fragmentées, photographiées, cruelles et violentes de pendus :

deux hommes vêtus de sortes de pyjamas rayés suspendus à des troncs d'arbres par les poignets, les mains liées. (...) Les épaules sont remontées et les têtes projetées en avant. Malgré la mauvaise qualité des photos, on peut voir que les visages des suppliciés sont tordus par la souffrance (ibid.:124)

et quelques lignes plus loin « un homme en uniforme d'officier, à la culotte de cavalerie bouffante au-dessus de bottes soigneusement cirées » (ibid.) matraque en main attendant que le supplicié à terre manifeste un peu de vie pour mieux le matraquer à nouveau, 
comme Novelli, lui-même battu à mort à son arrivée à Dachau, là où l'oncle de Quignard, Jean Bruneau (le fils de Charles), a été lui aussi déporté septembre 1944 à fin mai 1945. Fragments qui disent que ce qui distingue l'homme de l'animal, ce sont seulement sa conscience de la mort et sa cruauté. Quant à la guerre, elle suscite la narration, à penser comme retour de la prédation. Il y a quelque chose à dire, victoires ou catastrophes et à inscrire dans un temps historique, voire à muer en légende qui satisfasse l'ordre social et dans une langue qui elle-même est à sa source prédatrice :

Dans la guerre, chaque jour est non seulement narrable mais chaque jour devient narrateur. L'expérience qui y assaille est du "à-dire" qui ne cesse d'aller, de "une" en "une", de première page en première page, jour après jour, journal après journal, d'événement en événement, de vague en vague, de surgissement en surgissement. (Quignard, 2012 :205-206)

Après la guerre : Effondrement, destruction, désagrégation, brisure, ruines. Écriture sur des ruines, fragments qui ne cherchent pas à se ré-assembler: «Je ne pense pas par arguments, je pense toujours par images, par débris de rêves, par motions, par é-motions, par départs, par fugues, par extases, par scènes romanesques.» (Quignard, 2015 : 13) Figures revenantes de livre en livre comme les échos d'une voix perdue. Disparité des attaques, des mises en résonance :

Je pense qu'une ville neuve poussant sur un port renaissant anéanti, cela a destiné mes jours. Cela a influencé considérablement ce que j'écris. Les huit volumes parus de mon Dernier royaume c'est une immense reconstruction fragile sur des ruines plus toxiques et plus pulvérulentes que toutes les ruines jusque-là effondrées au cours de l'Histoire. (Quignard, $2013: 9$ )

Exemple : Le 10 juillet 2014, dans la cathédrale de Coutances, Quignard lit quelques pages d'une conférence qui va constituer « Les Ruines de Port-Royal », la première partie de Sur l'idée d'une communauté de solitaires. Jean-François Détrée est à l'orgue.

Je vais jouer la ligne mélodique en la mineur que je jouais en boucle au cours du mois de janvier 1997. C'est le plan, non plus titubant, mais lancinant de ce Dernier Royaume. C'est la vague qui sans cesse revient et, sur sa crête, éclate. Bien sûr, c'est une Allemande. C'est une Allemande de Rameau que j'ai follement aimé jouer pendant tous les mois de 1997 qui suivirent. (Quignard, 2015 : 35)

Cette première partie fait revenir Georges de La Tour, les Pascal, Blaise et ses sœurs Gilberte et Jacqueline, Meaume le graveur, Sainte-Colombe, Pontchâteau, l'un des Solitaires de Port-Royal et quelques-unes des pièces musicales sur lesquelles Quignard a écrit certains de ses livres: John Blow, Venus et Adonis ; François Couperin, «Les Ombres errantes » et "Leçons de ténèbres"; Sainte-Colombe, "Les Pleurs » et Rameau, une "Allemande ». Puis un passage sur ce qui fait le cœur du livre, l'idée d'une communauté de solitaires et l'anéantissement de leur abbaye: «En 1711 Port-Royal fut rasée sur l'ordre du roi Louis XIV en sorte qu'il "n'y restât pas pierre sur pierre".» (ibid.: 30) En écho, en reprise au début de la seconde partie "Compléments aux ruines »: "J'ai une dette avec les ruines. » (ibid. : 39) et dans « Baraques du lycée en ruines », le chapitre V de cette seconde partie : "Je vais chanter ce qui est en ruines. Tout ce qui est en ruines est en moi comme un premier visage » (ibid. : 56) Enfin, dans le chapitre suivant « Il y a deux perdus », comme une vague, retour sur la destruction de Port-Royal:

Il y a deux perdus. Il y a un perdu irrécupérable - c'est le perdu qui s'est véritablement égaré dans les ruines. Et il y a un perdu qu'on retrouve en soulevant les pierres, en désarticulant les mots, en décomposant les symboles et en en recomposant les fragments. (...) Durant l'hiver 1711, quand on exhuma trois mille corps à la pioche, les loups, les chiens, les ragondins, les renards - les corbeaux, les mulots, les chats, les merles - cherchaient à les désarticuler (...). Puis tout fut rasé. 
Tel était l'ordre qu'avait enjoint le roi à Versailles et dont il suivait personnellement la réalisation. Aucune ruine, ni soupçon de ruine, tel était l'ordre que le Roi Soleil avait donné à tous ses mandataires. (...) Il y a des ruines sans ruines. C'est Le Havre après la Seconde Guerre (...). (ibid. : 61-63)

De même, reviennent des guerres sans nombre, toutes plus cruelles les unes que les autres. Pearl Harbour: "Le dimanche 7 décembre 1941, jour où les Chrétiens s'approchent de leur idole pour aimer Dieu, l'aube s'étant levée, 188 bombardiers arrivèrent sur la côte d'Hawaï. (...)» (Quignard, 2002 : 17) ; Hiroshima : «On vit ce ravage $\mathrm{du}$ progrès sur le visage des hommes dans l'après-midi qui suivit la destruction d'Hiroshima. Ils erraient dans la chaleur d'août. Ils ne comprenaient pas la nature des maux qui les frappaient.» (Quignard, 2012 : 267) Ou encore le 11 septembre 2001 : « Explosion de mort dans le ciel bleu, à l'œil nu, le mardi 11 septembre 2001, au-dessus de la ville de New-York. » (Quignard, 2002 : 83). Ailleurs, la Saint-Barthélemy. La guerre de 1870, les guerres civiles, les guerres de Vendée et la Terreur révolutionnaire de 1793. Le terrorisme. Et le souvenir revenant du Havre. Quignard, enfant, sur son tricycle :

Il me faut confesser que, quand j'entrais sur mon tricycle à toute allure dans le square Saint-Roch du Havre, je pénétrais en chantant à tue-tête dans le charnier de la Seconde Guerre mondiale que la municipalité avait fait couvrir de fleurs. (Quignard, 2012:32)

Dans ce chapitre des Désarçonnés, le souvenir est associé au massacre de Guernica : «Il resta plus de maisons et d'églises debout à Guernica, petite ville de Biscaye, en mai 1937 que dans le port du Havre, en 1944.» (ibid. : 33) Dans d'autres livres, ce souvenir revient sur l'enfance au temps des ruines: «Je jouais dans le square qui avait été édifié sur le charnier des marins. J'étudiais dans les baraquements ». (Quignard, $2013: 8$ ), retour qui appelle le souvenir de l'exode des siens : « Tous, tous sans exception, fuyaient les bombes à fragmentation des souvenirs. » (ibid. : 10); son père sauvegardant « une tasse à café [de] Giacommo Meyerbeer » (Quignard, 2015 : 58). Et dans ce livre, l'image de l'enfant sur son « tricycle de fer » est associée à l'écriture sur des ruines des quatorze tomes de son Dernier Royaume (dont Quignard donne les titres), ruines que sont pêle-mêle « des vieux pneus, des portes arrachées, des pauvres citations du sanskrit, des galets couverts de mazout, des barques crevées » (ibid.: 33) dans une grande diversité de modes de représentation. $\mathrm{Du}$ « je », d'une écriture qui ramène sans ses filets maints souvenirs personnels, Quignard dit la guerre dans des évocations qui paraissent être un tableau ou une image de rêve un peu lointain : «Derrière l'épaule du passeur qui pesait de tout son corps sur sa gaffe dans le bassin du Roi, au Havre, très loin, à la limite des nuages, on voyait les silhouettes des navires anglais qui menaçaient les côtes d'une guerre de cent années. » (Quignard, 2002 : 119) Ailleurs, la brièveté de la phrase, ses précisions chronologiques démontent les fausses vertus de la guerre, en disent la jouissance: «Les Anglais ont tellement aimé bombarder Le Havre, en 1694, en 1759, en 1801, en 1944.» (Quignard, 2011 : 9).

\section{À quoi bon la littérature?}

Dès lors, quel pouvoir pour la littérature ? A-t-elle une quelconque utilité ? Simon affirme avec force dans « Tradition et révolution » (in Calle-Gruber, 2009) qu'aucune œuvre d'art, aucun roman n'a eu d'influence sur le cours de l'histoire. Il refuse tout autant la position de l'écrivain engagé que celle de "maître à penser " parce qu'il n'y a pas de dimension morale à attendre d'une œuvre littéraire. Lui, Simon, n'écrit pas de romans «à thèses ". Simplement, chez lui, la guerre fonde le récit à l'image du compagnon d'armes de Georges 
dans $L a$ Route des Flandres, enfermé comme lui dans le wagon à bestiaux qui les transporte vers le camp de prisonniers, qui reçoit un coup de botte dans la joue, quelques gouttes de sang s'écoulent. Il aura quelque chose à dire.

Et pourtant. Quelle littérature? Mireille Calle-Gruber donne les clés de compréhension d'une scène du Jardin des Plantes (Celle-Gruber, 2011 : 155-162). Simon, emmené par Dora Maar, débarque dans un appartement parisien cossu sur les quais, en 1944, où a lieu la première lecture publique du Diable attrapé par la queue. Sont présents Leiris, Camus, Queneau, Sartre, Reverdy entre autres " espèce de volière bienséante et feutrée » (Simon, 2006 : 1151), bref «l'intelligenstia parisienne d'alors», dit Mireille Calle-Gruber. Simon détourne son regard et voit passer une colonne de camions transportant de jeunes soldats allemands allant vers le front de Normandie. Lui revient en mémoire les camions de tirailleurs africains carbonisés sous ses yeux en mai 1940. Tout en continuant à percevoir vaguement dans son dos «le brouhaha de volière, bruyant, pâmé » (ibid. : 1154), il pense : « Pauvres bougres, pauvres bougres, pauvres bougres, pauvres bougres...» (ibid.: 1154) Plus tard, il écrira à Mireille Calle-Gruber que c'est de son appartement qui accueillait un service de chiffrage de messages de la Résistance que sera transmise à Londres l'information qui conduira au bombardement de la colonne par la RAF.

Et pourtant. Ailleurs, Simon rapporte son expérience de prisonnier en Allemagne pendant la Seconde Guerre mondiale : «J'ai connu la faim extrême. Pourtant, n'importe quel livre m'était précieux. Il ne trompait pas ma faim, ne l'apaisait pas, ne me la faisait même pas oublier : il s'agissait d'autre chose.» (in Calle-Gruber, 2009: 152). On se rappelle Si c'est un homme; Levi, prêt à se laisser mourir, se remémorant quelques vers appris par cœur de L'Enfer de Dante, à la demande de son ami Jean. Et puis, Dostoïevski, Souvenirs de la maison des morts; Michel del Castillo enfant placé pendant la guerre dans une sorte de bagne pour enfants, lisant Dostoïevski; Jean-Paul Kauffmann, otage au Liban, craignant quotidiennement d'être exécuté, lisant la Bible : «Le temps parfois dételle. Lire dételle. » (Quignard, 2012 : 132) Simon dans son désir constant de saisir le monde :

Un monde même momentanément privé d'art (...) serait tout simplement aussi cauchemardesque qu'un immense camp de concentration fonctionnel et bien organisé où la nourriture serait abondante, le travail peu pénible, les couvertures chaudes, les toits des baraques étanches, et où croupiraient non plus des hommes mais des bêtes. (in Calle-Gruber, 2009 : 152)

Mais il ne s'agit pas de témoigner, ni d'une quelconque défense morale parce que l'art est asocial. Écrire est « asocial » comme tout art (Simon citant Élie Faure). Il n'y a pas d'art engagé. Écrire pour d'abord continuer à vivre, parce qu'écrire est un faire: «Je fais (je produis), donc je suis, besoin, me semble-t-il élémentaire et que ressent tout homme normal (...) » (Simon, 2012 : 76) C'est un travail. C'est une façon de tenir : « Écrire n'est pas vivre, c'est survivre. » (Quignard, 2012 : 53). Dans l'écriture, l'écrivain n'est pas dans une position de surplomb par rapport à la réalité. Bien au contraire, il est totalement impliqué et totalement soumis à la puissance du langage :

Mais S. l'interrompt de nouveau et dit qu'il est impossible à qui que ce soit de raconter ou de décrire quoi que ce soit d'une façon objective, que, sauf dans des traités scientifiques comme par exemple d'anatomie ou de mécanique ou de botanique (encore que ce serait à discuter), il n'existe pas de style neutre ou comme on l'a aussi prétendu d'écriture «blanche » ce qui revient d'une façon assez naïve à entretenir le mythe d'un romancier dieu présenté comme un observateur impassible au regard détaché, «le monde comme si je n'étais pas là pour le dire » ironise déjà Baudelaire, le journaliste disant qu'il ne parlait pas de roman mais de 
témoignages, et $\mathrm{S}$. dit que six témoins différents d'un événement en donneront de bonne foi six versions différentes (...) (Simon, 2006 : 1100)

\section{BIBLIOGRAPHIE}

CALlE-GRUBER, Mireille, dir. (2009). Les Triptyques de Claude Simon. Paris : PSN.

CAlle-GRUBer, Mireille (2011). Claude Simon. Une vie à écrire. Paris : Seuil.

CAlle-Gruber, Mireille \& Quignard, Pascal (2013). La Suite des chats et des ânes. Paris : Presses de la Sorbonne Nouvelle.

CALLE-GRUBER, Mireille, Moreno, Melina Balcázar, Coulet, Sarah-Anaïs \& Franz, Anaïs, dir. (2014). Claude Simon. Les Vies de l'archive. Dijon : Éditions universitaires.

QUIGNARD, Pascal (1994). L'Occupation américaine. Paris : Seuil.

QUIGNARD, Pascal (2000 ; 1995). La Haine de la musique. Paris : Calmann-Lévy.

QUIGNARD, Pascal (2002). Les Ombres errantes. Paris : Grasset.

QUIGNARD, Pascal (2005). Les Paradisiaques. Paris : Grasset.

QUIGNARD, Pascal (2009). « Postface. Ce que vous a apporté Claude Simon », in Mireille CalleGruber (dir.). Les Triptyques de Claude Simon. Paris : Presses de la Sorbonne Nouvelle, pp. 207-211. QUIGNARD, Pascal (2011). «Le bac du Hode», Revue 2017 \& plus, n 2. Le Havre. Revue municipale. QUIGNARD, Pascal (2012). Les Désarçonnés. Paris : Grasset.

QUIGNARD, Pascal (2013). « L'estuaire », Pascal Quignard, Une enfance havraise. Le Havre : L'écho des vagues.

QUIGNARD, Pascal (2014). Mourir de penser. Paris : Grasset.

QUIGNARD, Pascal (2014). « Tradition de la non-tradition. Sur la lecture que Maurice MerleauPonty a faite de Claude Simon en 1959, en 1960 et en 1961 » in CALLE-GRUBER, (2014).

QUIGNARD, Pascal (2015). Sur l'idée d'une communauté de solitaires. Paris : Arlea.

SIMON, Claude (1964). « Pour qui donc écrit Sartre » in Calle-Gruber (2009) pp. 47-152.

SIMON, Claude (1967). « Tradition et révolution » in Calle-Gruber (2009) pp. 157-161.

SIMON, Claude (2012). Quatre conférences. Paris : Minuit.

SIMON, Claude (2006). Euvres complètes. Paris : Gallimard, « Bibliothèque de la Pléiade », tome I.

SIMON, Claude (2013). Euvres complètes. Paris : Gallimard, « Bibliothèque de la Pléiade », tome II. 
SIMON, Claude, manuscrits de L'Acacia conservés à la Bibliothèque Jacques Doucet, Paris. Cote SMN 16.

\section{NOTES}

1. Les manuscrits de Claude Simon sont conservés à la Bibliothèque Jacques Doucet à Paris.

2. «Bibliothèque de philosophie », éd. Aubier, 1996.

\section{RÉSUMÉS}

Simon/Quignard: deux temps vécus de la guerre (l'un, la mort de son père sur le champ de bataille en 1914 et pour lui, la guerre au front en 39-40; l'autre, les ruines de la guerre à sa fenêtre et les souvenirs de famille) impliquent deux imaginaires de la guerre très différents. Pourtant tous deux, en en démontant les ressorts, en proposent une analyse proche : la guerre dans sa cruauté loin de toute héroïsation est la marque de l'humain. Ses images, dans leur puissance de destruction, sont l'un des ressorts de leur pratique, très différente cependant dans l'écriture de la fragmentation. Leur imaginaire de la guerre suggère le pouvoir de la littérature.

Simon/Quignard : two life periods during wars (for one, his father's death in 1914 on the battlefield and his own experience in 1939-40; for the other, the ruins and destructions seen from his window and the family stories) call two very differing fiction worlds. Nevertheless both, when deconstructing the war engines, are producing an analysis in similar terms: war's cruelty, far from the hero building story tale is the mark of humanity. Its images, in their power of destruction, are one of the springs of their writing practice, although so different when writing the fragmentation. Their imaginary of war suggests the power of literature.

\section{INDEX}

Mots-clés : guerre, mort, ruines, fragment, déconstruction

Keywords : war, death, ruins, fragment, deconstruction

\section{AUTEUR}

\section{AGNÈS COUSIN DE RAVEL}

Université de Paris VII

agnescousinderavel@yahoo.fr 